\title{
A Generalization of Kharitonov's Four-Polynomial Concept for Robust Stability Problems with Linearly Dependent Coefficient Perturbations
}

\author{
B. ROSS BARMISH, MEMBER, IEEE
}

\begin{abstract}
From a systems-theoretic point of view, Kharitonov's seminal theorem on stability of interval polynomials suffers from two fundamental limitations. First, the theorem only applies to polynomials with independent coefficient perturbations. Note that uncertainty in the physical parameters of a linear system typically results in dependent perturbations in the coefficients of the characteristic polynomial. Secondly, Kharitonov's theorem only applies to zeros in the left-half planemore general zero location regions are not accommodated. In view of this motivation, the main result of this paper is a generalization of Kharitonov's four-polynomial concept to the case of linearly dependent coefficient perturbations and more general zero location regions. To this end, a specially constructed scalar function of a scalar variable is instrumental to the robustness analysis.
\end{abstract}

\section{INTRODUCTION AND TERMINOLOGY}

$\mathrm{T}$ HE seminal theorem of Kharitonov [1] has provided much impetus for recent research on the so-called robust stability problem, e.g., see Barmish [2] for a review of the continuoustime case and Bose and Zeheb [3] for the discrete-time case. To motivate the type of stability problems under consideration, consider a linear system whose characteristic polynomial depends on some vector of underlying physical parameters $q \in \boldsymbol{R}^{p}$. For example, the components $q_{i}$ of $q$ might represent various mass loadings, spring constants, and coefficients of friction. Within this framework, the characteristic polynomial is of the form

$$
p(s, q) \doteq \sum_{i=0}^{n} a_{i}(q) s^{i}
$$

and it is apparent that as $q$ varies over some prescribed bounding set $Q \subset \boldsymbol{R}^{p}$, we obtain a family of polynomials $Q$. More precisely,

$$
\mathcal{P} \doteq\{p(\cdot, q): q \in Q\} .
$$

Given the setup above, the system designer often wants to know if the zeros of all polynomials in $\mathscr{P}$ lie interior to some prespecified region $D$ in the complex plane. That is, $D$ might represent a specification on the closed-loop poles of a dynamic system and a robust design is one which guarantees that $p(s, q)$ has all its zeros in $D$ for all $q \in Q$. When this condition is satisfied, we say that $\mathcal{Q}$ is $D$-stable. Note that this terminology is suitable for both continuous-time and discrete-time problems-D can be the left-half plane, the unit disk, or a wide variety of other

Manuscript received November 24, 1987; revised June 27, 1988. Paper recommended by Associate Editor, M. A. Shayman. This work was supported by the National Science Foundation under Grant ECS-8612948

The author is with the Department of Electrical and Computer Engineering, University of Wisconsin-Madison, WI 53706.

IEEE Log Number 8825594 regions motivated by considerations such as damping, degree of stability, etc.

The breakthrough of Kharitonov [1] provides a complete solution to one special case of the $\mathscr{D}$-stability problem. Namely, $D$ is taken to be the left-half plane and $P$ is a so-called interval polynomial family. More precisely, letting

$$
a(q) \doteq\left[a_{0}(q) a_{1}(q) \cdots a_{n}(q)\right]^{T}
$$

Kharitonov assumes that the set of possible coefficient variations

$$
Q \doteq\{a(q): q \in Q\}
$$

is an $(n+1)$-dimensional rectangle obtained from a lower bound and an upper bound on each individual polynomial coefficient. Subsequently, it is established that $P$ is $D$-stable if and only if four distinguished polynomials, chosen from the $2^{n+1}$ extremes, all have their zeros in the strict left-half plane. Hence, the stability problem for the interval polynomial family $\mathcal{Q}$ is reduced to four applications of the Routh-Hurwitz stability criterion; for a simplification of Kharitonov's criterion for low-order polynomials, see Anderson, Jury, and Mansour [5].

There are two important motivations for the considerable research effort following Kharitonov [1]. First, Kharitonov's assumption that $\mathcal{Q}$ is an $(n+1)$-dimensional rectangle severely inhibits application of the result to practical problems. This rectangularity assumption is tantamount to having independent coefficient perturbations. Hence, when a physical parameter $q$ enters into more than one coefficient, one either abandons Kharitonov's theorem or applies it to an overbounding rectangle $\mathcal{Q}^{+}$which contains $Q$. This latter alternative leads to conservative results because the set of polynomials associated with $Q$ may be stable but the set of polynomials associated with $\mathcal{Q}^{+}$may turn out to be unstable, e.g., see Barmish, Fu, and Saleh [4].

The second motivation for further research stems from Kharitonov's assumption that $\mathscr{D}$ is the left-half plane. Even when $\mathbb{Q}$ is an $(n+1)$-dimensional rectangle, it is easy to construct $D$ regions (other than the left-half plane) for which $\mathfrak{D}$-stability of the set of $2^{n+1}$ extreme polynomials is not sufficient for $D$-stability of $P$. This point is established via counterexamples by a number of authors, e.g., see Bose and Zeheb [5] and Cieslik [6] for the case when $\mathbb{D}$ is the unit disk (discrete-time systems).

These counterexamples and the difficulties encountered treating dependent coefficient perturbations has driven much of the recent research in this area. Perhaps the strongest post-Kharitonov result to date is the edge theorem of Bartlett, Hollot, and Lin [7]; see also Fu and Barmish [8] for a refinement of the edge theorem. Bartlett, Hollot, and Lin assume that $D$ is simply connected and coefficient perturbations are polytopic. This polytopic requirement means that $\beta$ can be expressed as the convex hull of finitely 
many generating polynomials $p_{1}(s), p_{2}(s), \cdots, p_{l}(s)$. That is,

$$
\odot=\operatorname{conv}\left\{p_{1}(s), p_{2}(s), \cdots, p_{l}(s)\right\} .
$$

This assumption on $P$ is weaker than Kharitonov's rectangularity assumption because it allows for linearly dependent coefficient perturbations. To illustrate one of the many possible ways [2] arises, suppose that each physical parameter $q_{i}$ is restrained to an interval $\left[q_{i}^{-}, q_{i}^{+}\right]$. Then the bounding set $Q$ has at most $2^{p}$ extreme points. Letting

$$
q^{i} \doteq\left[q_{1}^{i} q_{2}^{i} \cdots q_{p}^{i}\right]^{T}
$$

denote the $i$ th such extreme point, note that

$$
q_{j}^{i}=q_{j}^{-} \text {or } q_{j}^{+}
$$

for $j=1,2, \cdots, p$, and moreover, the associated generating polynomial for the polytope $\odot$ is given by

$$
p_{i}(s)=\sum_{j=0}^{n} a_{j}\left(q^{i}\right) s^{j} .
$$

With $\odot$ being a polytope and the assumption of simple connectedness on $\mathscr{D}$, Bartlett, Hollot, and Lin [7] prove that $\mathscr{P}$ is $\mathscr{D}$-stable if and only if the set of exposed edges of $\mathcal{P}$ are $D$-stable. Hence, it is sufficient to show that for each pair $i, j \in\{1,2, \cdots, l\}$, the polynomial

$$
p_{i j}(s, \lambda) \doteq \lambda p_{i}(s)+(1-\lambda) p_{j}(s)
$$

is $D$-stable for all $\lambda \in[0,1]$. This result is important because the associated computational test for stability only involves a oneparameter sweep. This can be readily accomplished by viewing (1 - $\lambda) / \lambda$ as a variable parameter $k$ and generating a root locus diagram for $p_{i}(s)+k p_{j}(s)$. Now, one simply checks if this root locus remains entirely within the region $D$.

A number of authors provide various solutions and alternatives to the $\lambda$ sweeping problem above for special cases of interest. For example, when $D$ is the left-half plane, a certain type of eigenvalue test can be performed to ascertain $D$-stability of $p_{i j}(s$, $\lambda)$ for all $\lambda \in[0,1]$; see Bialas [9] and Fu and Barmish [10]. When $P$ corresponds to a low-order interval polynomial family with $D$ being the unit disk (discrete-time), the results of Kraus, Anderson, Jury, and Mansour [11] indicate that for polynomials of degree 5 or less, it suffices to simply check the $\mathscr{D}$-stability of the extreme polynomials $p_{i}(s)$ and various supplementary polynomials which lie on the edges. Finally, for the special case when $P$ is an interval polynomial family which depends on a single parameter and $\mathfrak{D}$ is the unit disk, $D$-stability can be ascertained via determinant-type calculations; see Bose, Jury, and Zeheb [12].

More recently, some additional alternatives to the $\lambda$ sweep have appeared. Eigenvalue tests have been developed for discrete-time systems which are quite similar in spirit to those given in Bialas [9] and $\mathrm{Fu}$ and Barmish [10] for continuous-time systems; see Bartlett and Hollot [13] and Ackermann and Barmish [14]. The work of Zeheb [15] allows for more general $D$ regions-only simple connectedness of $\mathscr{D}$ is assumed. Instead of a $\lambda$ sweep, Zeheb performs a sweep involving a scalar variable used in a parametric representation of the boundary of $D$. A similar boundary sweep is also the takeoff for the robust stability analysis of Saridereli and Kern [16]. Instead of having to deal with the combinatories of edges, their technique requires solution of a small linear program at each point along the boundary of $\mathfrak{D}$. This line of attack is also provided by Vicino [17].

In this paper, our main result is a generalization of Kharitonov's four-function concept to the case of linearly dependent coefficient perturbations. We also begin by sweeping the boundary of $D$ but unlike Saridereli and Kern [16], we avoid having to solve a linear program at each boundary point. To this end, we construct a real continuous scalar function $H(\delta)$ of a real variable $\delta$ and prove that $\boldsymbol{Q}$ is $D$-stable if and only if for each $\delta \in R, H(\delta)$ $>0$; see Section III. Henceforth, we refer to $H(\delta)$ as a robust stability testing function. From an implementation point of view, it is argued that our positivity test on $H(\delta)$ need only be carried out for $\delta$ in a bounded precomputable interval $\Delta$.

Some important points to note about this result are as follows.

1) For the special case when $\mathcal{P}$ is an interval polynomial family and $\mathfrak{D}$ is the left-half plane, the four Kharitonov polynomials fall out immediately from $H(\delta)$; see Section IV. This provides unity with the Kharitonov theory.

2) As the number of parameters $q_{i}$ increase, the previously discussed $D$-stability results involving the pairwise combinations $p_{i j}(s, \lambda)$ all require enormous computational effort. For example, if there are eight physical parameters $q_{i}$, then $P$ has 256 generating polynomials leading to 32640 pairwise combinations $p_{i j}(s, \lambda)$ to be considered. When the number of $q_{i}$ increases to 11 , the number of pairwise combinations $p_{i j}(s, \lambda)$ increases to 2096128 . More generally, if there are $p$ physical parameters $q_{i}$, the number of pairwise combinations which result is given by

$$
N_{P W}=2^{2 p-1}-2^{p-1} \text {. }
$$

In short, as $p$ increases, one must contend with a combinatoric explosion in $N_{P W}$. In contrast, the theory in this paper does not require separate calculations (such as a root locus or one of the other alternatives discussed above) for each exposed edge of $P$. The function $H(\delta)$ is generated using only the extreme points of P.

3) The theory presented here accommodates rather general regions $\mathfrak{D}$. For example, in Section $V$, we consider an example with a dominant pole specification of the following sort. The designer wants two closed-loop poles within a radius of 1.0 from $-2 \pm j$ and the remaining closed-loop poles with real part less than -5 .

4) It is felt that the approach described here for linearly dependent coefficient perturbations suggests lines of attack for more general classes of coefficient perturbations. This point is briefly discussed in Section V.

Finally, to complete this overview of related literature, it should be mentioned that there have also been considerable research developments involving the so-called $l^{2}$ uncertainty description, e.g., see Bhattacharyya [18] for a detailed exposition.

\section{Motivation: The Special Case of Interval Polynomials}

Before presenting the main results in Sections III and IV we use common sense considerations to derive a robust stability testing function $H(\delta)$ for the special case of interval polynomials, i.e., the set of possible coefficient variations $\mathcal{Q}$ is an $(n+1)$-dimensional rectangle. This simple five-step derivation provides motivation for the type of result we seek for the more general polytope problem. At this lower level of generality, it is easy to see how Kharitonov's four polynomials fall out from $H(\delta)$. In Section IV of this paper, it will be shown that our more general formula for $H(\delta)$ degenerates into the same formula obtained here.

\section{A. Step 1: Description of an Interval Polynomial Family $\odot$}

We consider the polynomial $p(s, q)$ as in (1) and obtain an (n $+1)$-dimensional rectangle for the set of coefficients $Q$ by taking

$$
\begin{gathered}
q=\left[q_{0} q_{1} \cdots q_{n}\right]^{T} \in R^{n+1}, \\
a_{i}(q)=q_{i}
\end{gathered}
$$

for $i=0,1,2, \cdots, n$ and the bounding set $Q$ for $q$ to be the $(n+$ 1)-dimensional rectangle

$$
Q=\left\{q \in R^{p}: q_{i}^{-} \leq q_{i} \leq q_{i}^{+} ; \quad i=0,1, \cdots, n\right\}
$$


where the coefficient bounds $q_{i}^{-}$and $q_{i}^{+}$are prescribed a priori. Hence, a typical polynomial $p(s, q)$ in $\odot$ looks like

with

$$
p(s, q)=\sum_{i=0}^{n} q_{i} s^{i}
$$

$$
q_{i}^{-} \leq q_{i} \leq q_{i}^{+}
$$

for $i \in\{0,1,2, \cdots, n\}$.

Finally, to avoid trivialities, we assume that $\odot$ contains at least one $\mathfrak{D}$-stable polynomial.

\section{B. Step 2: Description of the Four Kharitonov Polynomials}

Using the notational setup above, the four Kharitonov polynomials are

$$
\begin{aligned}
& K_{1}(s)=q_{0}^{-}+q_{1}^{-} s+q_{2}^{+} s^{2}+q_{3}^{+} s^{3}+q_{4}^{-} s^{4}+q_{5}^{-} s^{5}+q_{6}^{+} s^{6}+\cdots ; \\
& K_{2}(s)=q_{0}^{+}+q_{1}^{+} s+q_{2}^{-} s^{2}+q_{3}^{-} s^{3}+q_{4}^{+} s^{4}+q_{5}^{+} s^{5}+q_{6}^{-} s^{6}+\cdots ; \\
& K_{3}(s)=q_{0}^{+}+q_{1}^{-} s+q_{2}^{-} s^{2}+q_{3}^{+} s^{3}+q_{4}^{+} s^{4}+q_{5}^{-} s^{5}+q_{6}^{-} s^{6}+\cdots ; \\
& K_{4}(s)=q_{0}^{-}+q_{1}^{+} s+q_{2}^{+} s^{2}+q_{3}^{-} s^{3}+q_{4}^{-} s^{4}+q_{5}^{+} s^{5}+q_{6}^{+} s^{6}+\cdots
\end{aligned}
$$

\section{Step 3: The Generators for the Interval Polynomial Family} P form

There are at most $2^{n+1}$ generating polynomials ${ }^{1}$ for $\mathcal{P}$ of the

$$
p_{i}(s)=a_{n} s^{n}+a_{n-1} s^{n-1}+a_{n-2} s^{n-2}+\cdots+a_{2} s^{2}+a_{1} s+a_{0}
$$

with $a_{j}=q_{j}^{-}$or $q_{j}^{+}$for $j=0,1,2, \cdots, n$.

\section{Step 4: Bounding $p(s, q)$ for $s=j \delta$}

A number of authors (see, for example, Dasgupta and Bhagwat [19] and Yeung and Wang [20]) have noticed that a distinguishing feature of the Kharitonov polynomials is that for $s=j \delta$ with $\delta \in$ $\boldsymbol{R}$, they provide the sharpest possible bounds on the real and imaginary parts of $p(j \delta, q)$. More precisely, for $s=j \delta$ and $\delta \in$ $\boldsymbol{R}$, we introduce the following notation for the real and imaginary parts of the Kharitonov polynomials:

$$
\begin{aligned}
& R^{-}(\delta) \doteq \operatorname{Re} K_{1}(j \delta) ; \\
& R^{+}(\delta) \doteq \operatorname{Re} K_{2}(j \delta) ; \\
& I^{-}(\delta) \doteq \operatorname{Im} K_{3}(j \delta) ; \\
& I^{+}(\delta) \doteq \operatorname{Im} K_{4}(j \delta) .
\end{aligned}
$$

To illustrate, for $n=5$, we obtain

$$
\begin{aligned}
& R^{-}(\delta)=q_{4}^{-} \delta^{4}-q_{2}^{+} \delta^{2}+q_{0}^{-} ; \\
& R^{+}(\delta)=q_{4}^{+} \delta^{4}-q_{2}^{-} \delta^{2}+q_{0}^{+} ; \\
& I^{-}(\delta)=q_{5}^{-} \delta^{5}-q_{3}^{+} \delta^{3}+q_{1}^{-} \delta ; \\
& I^{+}(\delta)=q_{5}^{+} \delta^{5}-q_{3}^{-} \delta^{3}+q_{1}^{+} \delta .
\end{aligned}
$$

Now, it is easy to verify that for $\delta \geq 0$, the sharpest possible bounds for the real and imaginary parts of polynomials $p(s, q)$ in $Q$ are given by

$$
R^{-}(\delta) \leq \operatorname{Re} p(j \delta, q) \leq R^{+}(\delta)
$$

'Notice that there are less than $2^{n+1}$ distinct generators when some of the $q_{i}^{-}$and $q_{i}^{+}$are equal.

$$
I^{-}(\delta) \leq \operatorname{Im} p(j \delta, q) \leq I^{+}(\delta)
$$

and for $\delta<0$, the bounds become

$$
\begin{aligned}
R^{-}(\delta) & \leq \operatorname{Re} p(j \delta, q) \leq R^{+}(\delta) ; \\
I^{+}(\delta) & \leq \operatorname{Im} p(j \delta, q) \leq I^{-}(\delta) .
\end{aligned}
$$

\section{E. Step 5: The Stability Testing Function $H(\delta)$}

To obtain the desired robust stability testing function $H(\delta)$, we need two observations.

Observation 1: With $\mathcal{D}$ taken to be the left-half plane, the interval polynomial family $\mathcal{P}$ is $\mathscr{D}$-stable if and only if

$$
p(j \delta, q) \neq 0
$$

for all $\delta \in \boldsymbol{R}$ and all $q \in Q$. That is, since $\mathcal{P}$ contains at least one $D$-stable polynomial and the zeros of $p(s, q)$ vary continuously with $q, \mathcal{D}$-stability is guaranteed if and only if, for all $q \in Q, p(s$, $q$ ) does not vanish on the boundary of $D$-in this case, the boundary of $D$ is the imaginary axis. This type of boundary sweeping condition is used frequently in the literature, e.g., see Fam and Meditch [21], Argoun [22], and Hertz, Jury, and Zeheb [23].

Observation 2: In view of $\mathfrak{D}$-stability condition (12) and the rectangular bound above for $p(s, q)$ (its real and imaginary part), it follows that $P$ is $D$-stable if and only if for each $\delta \geq 0$, at least one of the four conditions $R^{-}(\delta)>0$ or $R^{+}(\delta)<0$ or $I^{-}(\delta)$ $>0$ or $I^{+}(\delta)<0$ holds, and, for each $\delta<0$, at least one of the following four conditions $R^{-}(\delta)>0$ or $R^{+}(\delta)<0$ or $I^{+}(\delta)>0$ or $I^{-}(\delta)<0$ holds. Equivalently, if we define

$H(\delta) \doteq \begin{cases}\max \left\{R^{-}(\delta),-R^{+}(\delta), I^{-}(\delta),-I^{+}(\delta)\right\} & \text { if } \delta \geq 0 \\ \max \left\{R^{-}(\delta),-R^{+}(\delta),-I^{-}(\delta), I^{+}(\delta)\right\} & \text { if } \delta<0\end{cases}$

it now follows that $\mathcal{Q}$ is $D$-stable if and only if

$$
H(\delta)>0
$$

for all $\delta \in \boldsymbol{R}$. Hence, for this special case, we have derived a robust stability testing function whose positivity is both necessary and sufficient for D-stability. It is this concept which we now generalize.

\section{The Main Results}

In this section, the main objective is to generalize the definition of $H(\delta)$ given for interval polynomials to accommodate more general polytopes of polynomials and more general $D$ regions. To this end, we introduce our two main assumptions.

Assumption 1: $P$ is a polytope as in (2) containing at least one D-stable polynomial. ${ }^{2}$

Assumption 2: $\mathscr{D}$ is the union of a finite number, call it $n_{\mathfrak{D}}$, of pathwise connected regions in the complex plane. We temporarily assume (for simplicity of notation) that $n_{\mathfrak{D}}=1$ and describe the extension for $n_{D}>1$ following the exposition of the main result in Section III. ${ }^{3}$

\section{A. Notation for Two Boundary Sweeping Functions}

In this subsection, we introduce two boundary sweeping functions, $\Phi_{\mathcal{D}}(\delta)$ and $\Phi_{\Gamma}(\rho)$. For various choices of $\mathcal{D}$, the first of these functions, $\Phi_{\mathscr{D}}$, is exploited in a number of recent papers on D-stability and related polynomial problems, e.g., see Fam and

${ }^{2}$ In fact, for the problem to be meaningful, there is no loss of generality in assuming that the generating polynomials $p_{1}(s), p_{2}(s), \cdots, p_{l}(s)$ are $D$-stable.

${ }^{3}$ The theory in this paper can also be used to determine if $\odot$ has a prescribed number of zeros in each connected component of $D$. To handle this case, we need only assume that there is at least one polynomial in $\sigma$ satisfying this given specification. 
Meditch [21], Zeheb [15], and Vicino [17]. The second of these functions, $\Phi_{\Gamma}$, is new and will be seen to play an important role in the construction of $H(\delta)$. Since $\Phi_{\mathscr{D}}$ and $\Phi_{\Gamma}$ will be nonuniquely defined, it is important to note that the main result to follow is independent of the specific choice of these functions subject to the stated restrictions. From the point of view of numerical computation, however, it will be seen that choosing $\Phi_{\Gamma}$ to be piecewise linear turns out to be quite convenient.

\section{B. The Function $\Phi_{\mathfrak{D}}(\delta)$}

We adopt the notation $\Phi_{\mathcal{D}}: R \rightarrow \partial D$ to denote a continuous mapping of the scalar variable $\delta$ onto the boundary of $D$, e.g., if $D$ is the half plane described by $\operatorname{Re} s \leq-\sigma$ for some $\sigma \geq 0$, then

$$
\Phi_{\mathscr{D}}(\delta)=-\sigma+j \delta
$$

will suffice and if $\mathfrak{D}$ is a disk centered at $s=\alpha+j \beta$, we can use

$$
\Phi_{\mathcal{D}}(\delta)= \begin{cases}(\alpha+\cos 2 \pi \delta)+j(\beta+\sin 2 \pi \delta) & \text { if } 0 \leq \delta \leq 1 ; \\ 0 & \text { otherwise }\end{cases}
$$

\section{The Function $\Phi_{\Gamma}(\rho)$}

Let $\Gamma$ be any region in the complex plane (chosen by the user) which has a continuous boundary $\partial \Gamma$ and contains the origin $s=0$ in its interior. We adopt the notation $\Phi_{\Gamma}:[0,1] \rightarrow \partial \Gamma$ to denote a continuous mapping of a scalar variable $\rho$ onto the boundary of $\Gamma$. For example, one simple choice would be to take $\Gamma$ to be the unit disk and

$$
\Phi_{\Gamma}(\rho)=\cos \pi \rho+j \sin 2 \pi \rho
$$

for $\rho \in[0,1]$. Note that this choice is valid for both continuoustime and discrete-time systems.

\section{Remarks About $\Phi_{\mathscr{D}}$ and $\Phi_{\Gamma}$}

The important point to note is that the user of the theorem to follow does not need to generate closed-form expressions for $\Phi_{D}(\delta)$ and $\Phi_{\Gamma}(\rho)$. The mechanization of the D-stability criterion in the theorem to follow simply involves sweeping the complex variable $s$ along the boundaries of $\partial D$ and $\partial \Gamma$. That is, one simply takes $\Phi_{\mathscr{D}}(\delta)=s$ when $s$ is being swept over the boundary of $D$ and $\Phi_{\Gamma}(\rho)=s$ when $s$ is being swept over the boundary of $\Gamma$. Hence, in numerical computations, we do not need to have explicit formulas for $\Phi_{\mathscr{D}}(\delta)$ and $\Phi_{\Gamma}(\rho)$. We simply view $\Phi_{\mathscr{D}}(\delta)$ and $\Phi_{\Gamma}(\rho)$ as symbolic notations.

\section{E. Generation of the Robust Stability Testing Function $H(\delta)$}

As a preliminary step, we introduce the following inner product notation. Given two complex numbers $z_{1}$ and $z_{2}$, let

$$
\left\langle z_{1}, z_{2}\right\rangle \doteq \operatorname{Re} z_{1} \cdot \operatorname{Re} z_{2}+\operatorname{Im} z_{1} \cdot \operatorname{Im} z_{2} .
$$

Then, for each fixed pair $(\rho, \delta) \in[0,1] \times R$, let

$$
h(\rho, \delta) \doteq \min _{i \leq l}\left\langle\Phi_{\Gamma}(\rho), p_{i}\left(\Phi_{\mathfrak{D}}(\delta)\right)\right\rangle .
$$

Now, for each fixed $\delta \in \boldsymbol{R}$, let $\rho^{*}(\delta)$ denote any maximizer of $h(\rho, \delta)$ with respect to $\rho \in[0,1]$. Then we define

$$
H(\delta)=h\left(\rho^{*}(\delta), \delta\right)
$$

or equivalently,

$$
H(\delta)=\max _{\rho \in[0,1]} h(\rho, \delta)
$$

\section{F. Theorem (See the Appendix for Proof)}

Consider the polytope of polynomials $\mathcal{P}$ and the region $\mathscr{D}$ satisfying Assumptions 1 and 2 . Then $P$ is $D$-stable if and only if for each $\delta \in \boldsymbol{R}$,

$$
H(\delta)>0 .
$$

\section{G. Extension for $n_{\mathfrak{D}}>1$}

Recalling the temporary assumption that $n_{\mathbb{D}}=1$, an examination of the proof in Appendix A readily reveals that when $n_{\mathbb{D}}>1$, the test for $D$-stability of $\mathcal{Q}$ is trivially modified as follows. One generates a separate $H(\delta)$ function for each of the components of $D-n_{\mathbb{D}}$ of them in this case. Subsequently, $D$-stability of $P$ is guaranteed if and only if all of these functions satisfy the positivity requirement in the theorem above. This is illustrated in Section V via an example.

\section{$H$. Restriction of the $\delta$ Sweep to a Bounded Interval $\Delta$}

Since the set of polynomials coefficients associated with $\mathcal{P}$ is compact, the zeros of polynomials in $\odot$ lie in a bounded subset of the complex plane. The consequence of this fact is that when $D$ is unbounded (for example, when $D$ is a half plane), it is not necessary to perform a sweep over the entire boundary of $D$ in testing for positivity of $H(\delta)$. By computing a crude bound for the zeros of polynomials in $\odot$, we can restrict the boundary sweep to a bounded subset of $\partial \mathscr{D}$. Equivalently, the $\delta$-sweep can be restricted to a bounded interval $\Delta \subset \boldsymbol{R}$. For example, given a polynomial

$$
p(s)=\sum_{i=0}^{n} a_{i} s^{i}
$$

it is well known (see, for example, Marden [24]) that all the zeros of $p(s)$ are interior to a circle of radius

$$
r \doteq 1+\max \left\{\left|a_{i} / a_{n}\right|: \quad i=0,1,2, \cdots, n-1\right\} .
$$

Hence, for a polytope of polynomials $\rho$ as in (2) whose $i$ th generator is given by (3), it is easy to verify that any polynomial in $\rho$ must have its zeros interior to a circle of radius

$$
R \doteq 1+\frac{\max \left\{\left|a_{j}\left(q^{i}\right)\right|: i=1,2, \cdots, l ; j=0,1, \cdots, n-1\right.}{\min \left\{\left|a_{n}\left(q^{i}\right)\right|: i=1,2, \cdots, l\right\}}
$$

It now follows that the $\delta$ sweep can be restricted to the intersection of the sphere of radius $R$ and the boundary of $D$. For example, if $\mathfrak{D}$ is the left-half plane, then with $\Phi_{\mathfrak{D}}(\delta)=j \delta$, one need only consider $\delta \in \Delta=[-R, R]$. It is also easy to show that $H(\delta) \rightarrow+\infty$ as $|\delta| \rightarrow \infty$. Hence, in practice, it is usually not necessary to compute a priori bounds for the $\delta$ sweep. Of course, if $D$ is already bounded (for example, if $D$ is the unit disk), the bounding procedure above is not necessary. Finally, it is also worth noting that the sweep can also be reduced when one part of the boundary of $D$ is the complex conjugate of another part of the boundary. For example, when $\mathscr{D}$ is the left-half plane and $\Phi_{\mathscr{D}}(\delta)$ $=j \delta$, one need only consider $\delta \geq 0$.

\section{Kharitonov Polynomials as a Special Case and COMPUTATIONAL Simplification}

In this section, we consider the special case of the theorem above which results when $\Gamma$ is taken to be the $l^{1}$ unit ball, i.e.,

$$
\Gamma=\left\{\gamma_{1}+j \gamma_{2}:\left|\gamma_{1}\right|+\left|\gamma_{2}\right| \leq 1\right\} .
$$


There are two reasons for considering this special case. First, when $P$ is an interval polynomial family and $\mathscr{D}$ is the left-half plane, this choice of $\Gamma$ is shown to lead to the same robust stability testing function $H(\delta)$ which was obtained for the Kharitonov analysis in Section II. That is, we obtain a unification with the Kharitonov theory.

The second motivation for this choice of $\Gamma$ stems from computational considerations. Although the choice of $\Gamma$ is rather arbitrary as far as validity of the theorem is concerned, numerical computation is quite a different matter. When $\Gamma$ is the $l^{1}$ unit ball, we obtain a degree of simplification in the computation of $H(\delta)-$ even when $\mathcal{Q}$ and $D$ are unrestricted. For fixed $\delta$, it will be seen that $h(\rho, \delta)$ turns out to be a piecewise linear concave function of $\rho \in[0,1]$. This is seen to facilitate numerical computation of $\rho^{*}(\delta)$ and $H(\delta)$

\section{A. Step 1: Description of $\Phi_{\Gamma}(\rho)$ with $\Gamma$ as the $l^{1}$ Unit Ball}

It is straightforward to verify that the interval $[0,1]$ is mapped continuously onto the boundary of the $l^{1}$ unit ball by

$$
\Phi_{\Gamma}(\rho)= \begin{cases}(1-4 \rho)+j 4 \rho & \text { if } 0 \leq \rho \leq 1 / 4 \\ (1-4 \rho)+j(2-4 \rho) & \text { if } 1 / 4<\rho \leq 1 / 2 \\ (-3+4 \rho)+j(2-4 \rho) & \text { if } 1 / 2<\rho \leq 3 / 4 \\ (-3+4 \rho)+j(-4+4 \rho) & \text { if } 3 / 4<\rho \leq 1\end{cases}
$$

B. Step 2: Computation of $h(\rho, \delta)$ with Polytopic $\mathcal{P}$, General $D$, and $\Gamma$ being the $l^{I}$ Unit Ball

By substituting the expression for $\Phi_{\Gamma}(\rho)$ above into (13), we obtain

$$
h(\rho, \delta)= \begin{cases}h_{1}(\rho, \delta) & \text { if } 0 \leq \rho \leq 1 / 4 \\ h_{2}(\rho, \delta) & \text { if } 1 / 4<\rho \leq 1 / 2 \\ h_{3}(\rho, \delta) & \text { if } 1 / 2<\rho \leq 3 / 4 \\ h_{4}(\rho, \delta) & \text { if } 3 / 4<\rho \leq 1\end{cases}
$$

where, for fixed $\delta \in \boldsymbol{R}$, the functions $h_{i}(\rho, \delta)$ above are piecewise linear and concave with respect to $\rho \in[0,1]$ and given by

$$
\begin{array}{r}
h_{1}(\rho, \delta) \doteq \min _{i \leq l}\left\{(1-4 \rho) \operatorname{Re} p_{i}\left(\Phi_{\mathscr{D}}(\delta)\right)+4 \rho \operatorname{Im} p_{i}\left(\Phi_{\mathscr{D}}(\delta)\right)\right\} ; \\
h_{2}(\rho, \delta) \doteq \min _{i \leq l}\left\{(1-4 \rho) \operatorname{Re} p_{i}\left(\Phi_{\mathcal{D}}(\delta)\right)+(2-4 \rho) \operatorname{Im} p_{i}\left(\Phi_{\mathcal{D}}(\delta)\right)\right\} ; \\
h_{3}(\rho, \delta) \doteq \min _{i \leq l}\left\{(-3+4 \rho) \operatorname{Re} p_{i}\left(\Phi_{\mathscr{D}}(\delta)\right)\right. \\
\left.+(2-4 \rho) \operatorname{Im} p_{i}\left(\Phi_{\mathfrak{D}}(\delta)\right)\right\} ; \\
h_{4}(\rho, \delta) \doteq \min _{i \leq l}\left\{(-3+4 \rho) \operatorname{Re} p_{i}\left(\Phi_{\mathscr{D}}(\delta)\right)\right. \\
\left.+(-4+4 \rho) \operatorname{Im} p_{i}\left(\Phi_{\mathfrak{D}}(\delta)\right)\right\} .
\end{array}
$$

C. Step 3: Computation of $H(\delta)$ with Polytopic $P$, General D, and $\Gamma$ being the $l^{l}$ Unit Ball

Now, in accordance with $(15), h(\mathrm{R}, \delta)$ must be maximized with respect to $\rho \in[0,1]$. In view of Step 2, this leads to the formula

$$
H(\delta)=\max \left\{H_{1}(\delta), H_{2}(\delta), H_{3}(\delta), H_{4}(\delta)\right\}
$$

where

$$
H_{i}(\delta)=\max _{\rho \in\{(i-1) / 4,(i / 4)\}} h_{i}(\rho, \delta)
$$

for $i=1,2,3,4$.
Note that the generation of the scalar functions $H_{i}(\delta)$ above is quite straightforward and readily lends itself to computer-aided graphics. For fixed $\delta$, one can easily display the piecewise linear scalar function $h_{i}(\cdot, \delta)$ and the maximum value $H_{i}(\delta)$ can be simply picked off the graph. Subsequently, as $\delta$ varies, the graph of $h_{i}(\cdot, \delta)$ shifts and $H_{i}(\delta)$ is obtained as the upper envelope of this one-parameter family of functions.

\section{Step 4: Further Specialization-Kharitonov's Problem}

The main objective in this subsection is to compute a closed form for $H(\delta)$ for the special case when $\beta$ is an interval polynomial family, $D$ is the left-half plane, and $\rho$ is the $l^{1}$ unit ball-this is the problem considered by Kharitonov. It will be shown that our general formula for $H(\delta)$ degenerates into the same formula derived in Section III by common sense considerations.

Indeed, we take the generating polynomials $p_{i}(s)$ for $\beta$ as given in (2) and begin with the computation of $H_{1}(\delta)$. Now, for $\delta \geq 0$ and $\rho \in[0,1 / 4]$, we have

$$
\begin{aligned}
h_{1}(\rho, \delta) & =\min _{i \leq l}\left\{(1-4 \rho) \operatorname{Re} p_{i}\left(\Phi_{\mathcal{D}}(\delta)\right)+4 \rho \operatorname{Im} p_{i}\left(\Phi_{\mathfrak{D}}(\delta)\right)\right\} \\
& =(1-4 \rho) R^{-}(\delta)+4 \rho I^{-}(\delta) .
\end{aligned}
$$

Hence, it is immediately apparent that the maximum of $h_{1}(\rho, \delta)$ with respect to $\rho \in[0,1 / 4]$ is attained at either $\rho=0$ or $\rho=1 / 4$. That is, for $\delta \geq 0$,

$$
H_{1}(\delta)=\max \left\{R^{-}(\delta), I^{-}(\delta)\right\} .
$$

A similar calculation for $\delta<0$ results in

$$
H_{1}(\delta)=\max \left\{R^{-}(\delta), I^{+}(\delta)\right\} \text {. }
$$

The computations of $H_{2}(\delta), H_{3}(\delta)$, and $H_{4}(\delta)$ are performed in an identical manner and lead to

$$
\begin{aligned}
& H_{2}(\delta)= \begin{cases}\max \left\{-R^{+}(\delta), I^{-}(\delta)\right\} & \text { if } \delta \geq 0 ; \\
\max \left\{-R^{+}(\delta), I^{+}(\delta)\right\} & \text { if } \delta<0 ;\end{cases} \\
& H_{3}(\delta)= \begin{cases}\max \left\{-R^{+}(\delta),-I^{+}(\delta)\right\} & \text { if } \delta \geq 0 ; \\
\max \left\{-R^{+}(\delta),-I^{-}(\delta)\right\} & \text { if } \delta<0 ;\end{cases} \\
& H_{4}(\delta)= \begin{cases}\max \left\{R^{-}(\delta),-I^{-}(\delta)\right\} & \text { if } \delta \geq 0 ; \\
\max \left\{R^{-}(\delta),-I^{+}(\delta)\right\} & \text { if } \delta<0 .\end{cases}
\end{aligned}
$$

Finally, substituting the $H_{i}(\delta)$ into the expression for $H(\delta)$, we obtain

$$
\begin{aligned}
H(\delta) & =\max \left\{H_{1}(\delta), H_{2}(\delta), H_{3}(\delta), H_{4}(\delta)\right\} \\
& = \begin{cases}\max \left\{R^{-}(\delta),-R^{+}(\delta), I^{-}(\delta),-I^{+}(\delta)\right\} & \text { if } \delta \geq 0 ; \\
\max \left\{R^{-}(\delta),-R^{+}(\delta),-I^{-}(\delta), I^{+}(\delta)\right\} & \text { if } \delta<0\end{cases}
\end{aligned}
$$

which is identical to the $H(\delta)$ function obtained in Section II-A.

\section{NUMERICAL EXAMPLES}

In this section, two numerical examples are provided to illustrate the application of the main results.

\section{A. Example 1: Dominant Pole Problem}

We consider a perturbed polynomial of the form (1) given by

$$
p(s, q)=s^{3}+\left(10+q_{2}\right) s^{2}+\left(29+q_{1}\right) s+\left(30+q_{1}+q_{2}\right)
$$

and observe that the nominal polynomial $p(s, 0)$ has zeros at $s=$ -6 and $s=-2 \pm j$. To illustrate application of the theory, suppose that the objective is to guarantee that two of the zeros 
remain within circles of radii $\epsilon=1$ centered at $s=-2 \pm j$ and the third zero has real part $\leq-5$. We take uncertainty bounds of the form

$$
\left|q_{i}\right|<\bar{q}_{i}
$$

where $\vec{q}_{i} \geq 0$ for $i=1,2$. To further simplify, we take

$$
\bar{q}_{1}=\bar{q}_{2} \doteq \bar{q}
$$

and study the satisfaction of the zero location specification as a function of $\bar{q}$. That is, how large can $\bar{q}$ be before $D$-stability is lost? ${ }^{4}$

To begin the analysis, note that Assumptions 1 and 2 of Section III are satisfied because the $D$ region for this problem is the union of three pathwise connected sets (a half plane and two disks) and $p(s, 0)$ is $\mathfrak{D}$-stable. For various values of $\bar{q}$, calculations were subsequently performed as follows.

Four extreme polynomials were formed as prescribed by

2) The boundary sweeping function $\Phi_{D}$ for a half plane and two disks was taken as in Section III-B with $\sigma=5, \alpha=-2$, and $\beta=$ -1 .

3) The set $\Gamma$ was takển to be the $l^{1}$ unit ball.

4) A bounded interval $\Delta$ for the $\delta$ sweep was determined using the crude bound (17).

5) The robust stability testing function was generated in accordance with the recipe in Section IV and plotted.

The plots given in Figs. 1-3 indicate the $H(\delta)$ function for three representative values of the perturbation bound $\bar{q}$. By inspection, we see that $H(\delta)$ goes slightly negative $\bar{q}=0.40$ [see Fig. 1(a) and (b)] indicating that this value of $\bar{q}$ is near the marginal stability point. For illustrative purposes, also notice that $H(\delta)$ goes negative for $\bar{q}=5.0$ and $\bar{q}=10.0$ [see Fig. 2(a) and (b) and Fig. 3 (a) and (b)]-indicating a lack of $\mathfrak{D}$-stability. In order to find the maximum tolerable perturbation bound $\bar{q}_{\max }$, a number of additional runs were made for values of $\bar{q}$ between 0.25 and 5.0. It was concluded that

$$
\bar{q}_{\max } \approx 0.35 \text {. }
$$

\section{B. Example 2: Discrete-Time Interval Polynomial}

This example was previously considered in Bose, Jury, and Zeheb [12]. By generating the $H(\delta)$ function, we show that the perturbation bound $(\bar{q} \approx 0.47)$ obtained by these authors can be increased by more than a factor of two. Indeed, we take $D$ to be the unit disk and investigate the $D$-stability of the interval polynomial family described by

$$
p(s, q)=\left(5+q_{4}\right) s^{4}+\left(1+q_{3}\right) s^{3}+\left(1+q_{2}\right) s^{2}+\left(1+q_{1}\right) s+\left(1+q_{0}\right)
$$

with perturbation bound

$$
\left|q_{i}\right| \leq \bar{q}
$$

for $i=0,1, \cdots, 5$. The objective is to determine the largest value of $\bar{q}$ for which $D$-stability is guaranteed. The analysis proceeds in the same way as indicated in 1)-5) of Example 1. In Fig. 4, we see that for $\bar{q}=0.4, \bar{H}(\delta)$ remains positive and $D$ stability is therefore guaranteed. On the other hand, in Fig. 5, we see that for $\bar{q}=1.25, H(\delta)$ dips negative indicating the absence of D-stability. Finally, in Fig. 6, we show the marginal Dstability case. That is, for

$$
\bar{q}=\bar{q}_{\max } \approx 1.0,
$$

the minimum value of $H(\delta)$ is close to zero; we computed a minimum value of 0.0106 .

${ }^{4}$ It is important to note that the specification on the zeros of $p(s, q)$ is satisfied when $q=0$. This fact is important because the $\mathscr{D}$ region has three components.

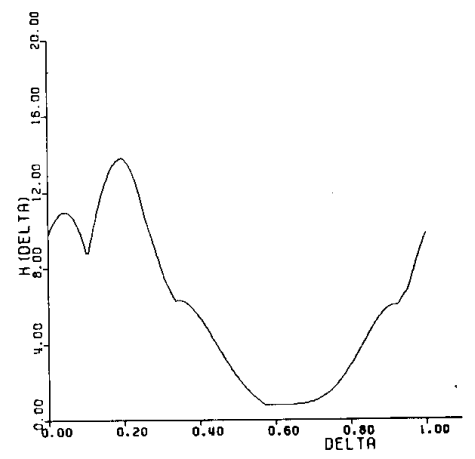

(a)

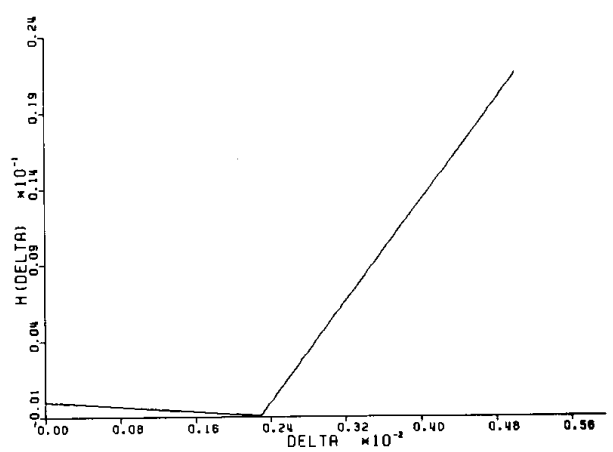

(b)

Fig. 1. (a) $H(\delta)$ for Example 1 with $\bar{q}=0.4$ (disk). (b) $H(\delta)$ for Example 1 with $\bar{q}=0.4$ (axis).

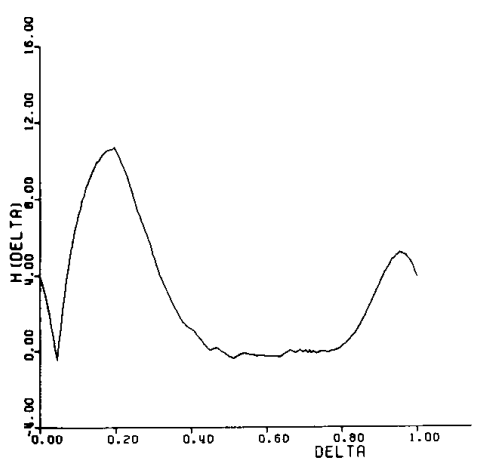

(a)

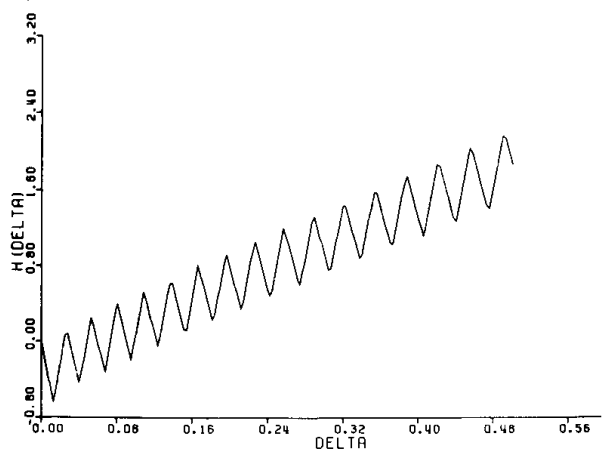

(b)

Fig. 2. (a) $H(\delta)$ for Example 1 with $\bar{q}=5.0$ (disk). (b) $H(\delta)$ for Example 1 with $\bar{q}=5.0$ (axis). 


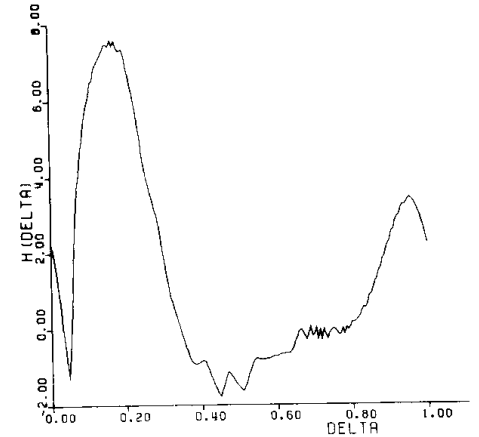

(a)

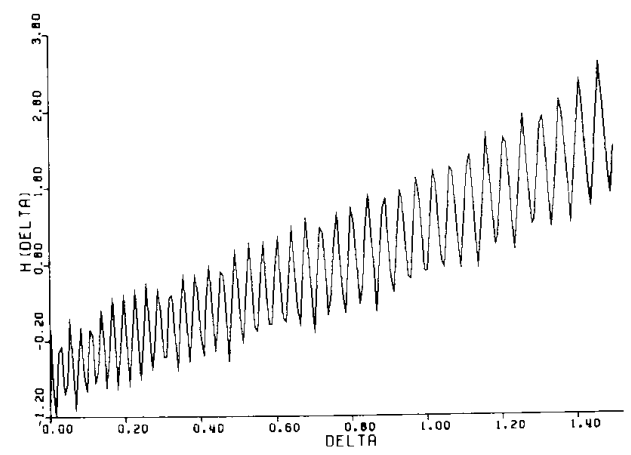

(b)

Fig. 3. (a) $H(\delta)$ for Example 1 with $\bar{q}=10.0$ (disk). (b) $H(\delta)$ for Example 1 with $\bar{q}=10.0$ (axis).

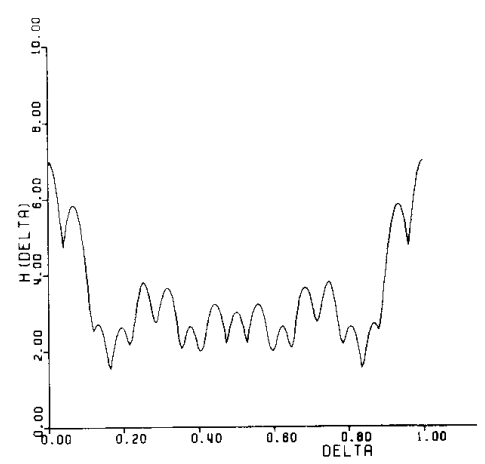

Fig. 4. $H(\delta)$ for Example 2 with $\bar{q}=0.4$.

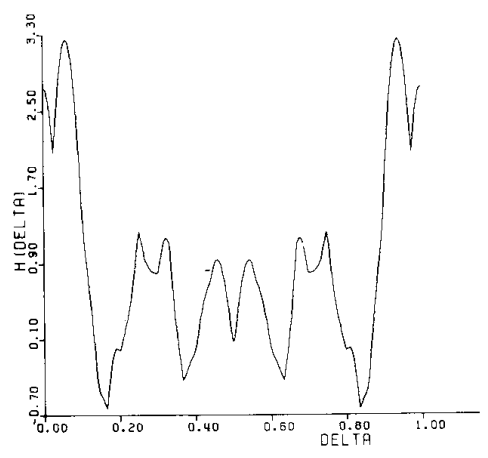

Fig. 5. $H(\delta)$ for Example 2 with $\bar{q}=1.25$.

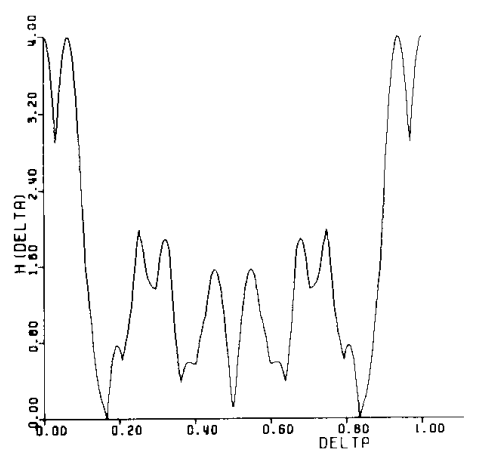

Fig. 6. $H(\delta)$ for Example 2 with $\bar{q}=1.0$.

\section{CONCLUSION}

To keep the concluding discussion simple, suppose $D$ is the strict left-half plane. Then this paper and a number of others all begin their technical analysis from the same starting point. Namely, under the stated assumptions, robust stability is guaranteed if and only if the point $s=0$ is excluded from the set of polynomial values

$$
\Omega(\omega)=\{p(j \omega, q): q \in Q\}
$$

for all frequencies $\omega \in \boldsymbol{R}$. This relationship between zero exclusion and robustness has been known for a long time, e.g., when the coefficients $a_{i}(q)$ are multilinear functions of $q$, the robustness problem is set up precisely in this manner in Zadeh and Desoer [25]. What fuels current research is the issue of computational tractability, i.e., are there classes of robustness problems for which the test for zero exclusion is reasonable to perform. The polytope structure provided here is one such example.

For the more general multilinear problem, Saeki [26] provides a sufficient condition for robust stability by working with the recent paper by de Gaston and Safonov [27], which provides an algorithmic approach for testing the zero exclusion condition. They provide a cutting hyperplane-type algorithm to ascertain whether or not $0 \in \Omega(\omega)$, and in a follow-up paper, Sideris and de Gaston [28] extend these ideas to handle the case when the $a_{i}(q)$ are polynomic functions of $q$.

The research presented here raises the possibility that for the multilinear coefficient perturbation problem, there may be an analytical test for stability, e.g., perhaps it is possible to generalize the definition of the robust stability testing function $H(\delta)$ so as to handle this case. To this end, note that the so-called mapping theorem in Zadeh and Desoer [25] can be used to prove that the smallest convex set containing the set of possible coefficients $Q$ is a polytope having generators given by (3); this is one of the main ideas in a paper by Saeki [26]. Hence, one is tempted to conjecture that in the multilinear case, the same formula (15) can be used for $H(\delta)$. Unfortunately, this conjecture is false; the conservatism associated with this convex hull approach was first recognized in de Gaston and Safonov [27] and in Barmish, Fu, and Saleh [4], a counterexample of the following sort is provided. All coefficients in $Q$ represent strictly stable polynomials but the convex hull of $\mathscr{Q}$ includes coefficient vectors associated with unstable polynomials. Hence, using the convex hull of $Q$ instead of $Q$ itself clearly introduces a degree of conservatism into the analysis. That is, positivity of $H(\delta)$ is sufficient but no longer necessary for $\mathfrak{D}$-stability.

APPENDIX: Proof of Theorem

Preliminaries: First, it should be observed that every polynomial in $\mathcal{P}$ is a convex combination of the form

$$
p(s, \lambda) \doteq \sum_{i=1}^{l} \lambda_{i} p_{i}(s)
$$


with

$$
\lambda \doteq\left[\lambda_{1} \lambda_{2} \cdots \lambda_{l}\right]^{T}
$$

in the unit simplex

$$
\Lambda \doteq\left\{\lambda \in \boldsymbol{R}^{\prime}: \lambda_{i} \geq 0 \text { for all } i \text { and } \sum_{i=1}^{l} \lambda_{i}=1\right\}
$$

The proof will be carried out in three steps. Note that the first step of the proof involves a rather standard connectivity argument which we include for the sake of completeness.

Step 1: We claim that $P$ is $D$-stable if and only if

$$
p\left(\Phi_{\mathfrak{D}}(\delta), \lambda\right) \neq 0
$$

for all $\delta \in R$ and all $\lambda \in \Lambda$. First, the necessity of condition (18) is trivial because if $p\left(\Phi_{\mathcal{D}}\left(\delta^{*}\right), \lambda^{*}\right)=0$ for some $\delta^{*} \in R$ and some $\lambda^{*} \in \Lambda$, then we have a contradiction to the $\mathscr{D}$-stability of $\mathcal{P}$; i.e., there is a polynomial in $\mathcal{P}$ having a zero outside the interior of $D$.

The sufficiency of condition (18) is also proved by contradiction. Indeed, suppose condition (18) holds but $P$ is not $D$-stable. Then for some $\lambda^{0} \in \Lambda$, the polynomial $p\left(s, \lambda^{0}\right)$ has at least one zero outside the interior of $D$. Now, we let $p_{\star}(s)$ denote any $D$ stable polynomial in $P$ (recall Assumption 1) and with $\alpha \in[0,1]$, we define the $\alpha$-parameterized family of polynomials in $\rho$ by

$$
f(s, \alpha) \doteq(1-\alpha) p_{*}(s)+\alpha p\left(s, \lambda^{0}\right)
$$

and notice that this family is contained within $P$. Moreover, $f(s, 0)=p_{*}(s)$ has all its zeros interior to $D$ and $f(s, 1)=p(s$, $\lambda^{0}$ ) has at least one zero outside the interior of $D$. Hence, by continuity of the zeros of $f(s, \alpha)$ with respect to $\alpha$, it follows that there exists some $\alpha^{*} \in(0,1]$ such that $f\left(s, \alpha^{*}\right)$ has a zero in $\partial \mathcal{D}$; say $s=z^{*} \in \partial \mathscr{D}$ is this zero. Now, choosing $\delta^{*} \in R$ such that $\Phi_{D}\left(\delta^{*}\right)=z^{*}$, and $\lambda^{*} \in \Lambda$ such that

$$
p\left(s, \lambda^{*}\right)=f\left(x, \alpha^{*}\right)
$$

for all $s$, it follows that

$$
p\left(\Phi_{\triangleright}\left(\delta^{*}\right), \lambda^{*}\right)=0
$$

which is the contradiction we seek.

Step 2: In this step of the proof, we use a separating hyperplane argument. To this end, for each $\delta \in R$ and each $\lambda \in \Lambda$, we break $p\left(\Phi_{D}(\delta), \lambda\right)$ into its real and imaginary parts

$$
p\left(\Phi_{\mathcal{D}}(\delta), \lambda\right)=\operatorname{Re} p\left(\Phi_{\mathcal{D}}(\delta), \lambda\right)++j \operatorname{Im} p\left(\Phi_{\mathscr{D}}(\delta), \lambda\right)
$$

and simply note that the satisfaction of $\mathfrak{D}$-stability requirement (18) is equivalent to the following condition. For each $\delta \in \Lambda$, either

$$
\operatorname{Re} p\left(\Phi_{\mathfrak{D}}(\delta), \lambda\right) \neq 0
$$

or

$$
\operatorname{Im} p\left(\Phi_{\mathfrak{D}}(\delta), \lambda\right) \neq 0 .
$$

Now, for each fixed $\delta \in R$, we define the polytope

$$
\begin{aligned}
\mathbf{\Omega}(\delta) & =\left\{p\left(\Phi_{\mathscr{D}}(\delta), \lambda\right): \lambda \in \Lambda\right\} \\
& =\operatorname{conv}\left\{p_{1}\left(\Phi_{\mathscr{D}}(\delta)\right), p_{2}\left(\Phi_{\mathcal{D}}(\delta)\right), \cdots, p_{l}\left(\Phi_{\mathscr{D}}(\delta)\right)\right\} .
\end{aligned}
$$

It now follows that the satisfaction of (19) and (20) is equivalent to the requirement that the origin in the complex plane can be separated from the polytope $\Omega(\delta)$ by a line. Hence, $\mathcal{P}$ is $D$-stable if and only if for each $\delta \in R$, there exists some nonzero vector $\eta$ $\doteq\left[\eta_{1} \eta_{2}\right]$ such that

$$
\eta_{1} \operatorname{Re} p\left(\Phi_{\mathcal{D}}(\delta), \lambda\right)+\eta_{2} \operatorname{Im} p\left(\Phi_{\mathfrak{D}}(\delta), \lambda\right)>0
$$

for all $\lambda \in \Lambda$.
Step 3: In this final step of the proof, we examine the ramifications of the separating hyperplane condition (21) for $\mathfrak{D}$ stability. The first point to note is that we restrict attention to $\eta \in$ $\partial \mathrm{B}$ because of the homogeneity with respect to $\eta$ on the left-hand side of (21).

The second point to note is that the D-stability condition (21) need not be checked for all $\lambda \in \Lambda$. That is, 0 can be separated from $\Omega(\delta)$ if and only if 0 can be separated from the set of extreme points of $\Omega(\delta)$. Hence, it follows that $\Phi$ is $D$-stable if and only if for each $\delta \in R$, there exists some $\eta \in \partial \Gamma$ such that

$$
\eta_{1} \operatorname{Re} p_{i}\left(\Phi_{D_{D}}(\delta)\right)+\eta_{2} \operatorname{Im} p_{i}\left(\Phi_{D_{D}}(\delta)\right)>0
$$

for $i \in\{1,2, \cdots, l\}$. Equivalently, $\mathcal{P}$ is $D$-stable if and only if for each $\delta \in R$, there exists some $\rho \in[0,1]$ such that

$$
\operatorname{Re} \Phi_{\Gamma}(\rho) \cdot \operatorname{Re} p_{i}\left(\Phi_{\mathscr{D}}(\delta)\right)+\operatorname{Im} \Phi_{\Gamma}(\rho) \cdot \operatorname{Im} p_{i}\left(\Phi_{\mathcal{D}}(\delta)\right)>0
$$

for $i \in\{1,2, \cdots, l\}$. Substituting for $h(\rho, \delta)$ above, we arrive at the following point: $P$ is $D$-stable if and only if for each $\delta \in R$, there exists some $\rho \in[0,1]$ such that

$$
h(\rho, \delta)>0 .
$$

The proof is now completed by re-expressing this condition in terms of the maximum of $h(\rho, \delta)$ with respect to $\rho \in[0,1]$. That is, $P$ is $D$-stable if and only if for each $\delta \in R$,

$$
0<h\left(\rho^{*}(\delta), \delta\right)=H(\delta) .
$$

The proof of the theorem is now complete.

\section{REFERENCES}

[1] V. L. Kharitonov, "Asymptotic stability of an equilibrium position of a family of systems of linear differential equations," Differential'nye Uraveniya, vol. 14, no. 11, pp. 1483-1485, 1978

[2] B. R. Barmish, "New tools for robustness analysis," in Proc. IEEE Conf. Decision Contr., Austin, TX, 1988.

[3] N. K. Bose and E. Zeheb, "Kharitonov's theorem and stability test of multidimensional digital filters," IEE Proc. $-G$, vol. 133 , no. 4, pp. $187-190,1986$

[4] B. R. Barmish, M. Fu, and S. Saleh, "Stability of a polytope of matrices: Counterexamples," IEEE Trans. Automat. Contr., vol. AC-33, pp. 569-572, June 1987.

[5] B. D. O. Anderson, E. I. Jury, and M. Mansour, "On robust Hurwitz polynomials," IEEE Trans. Automat. Contr., vol. AC-32, pp. 909912 , Oct. 1987.

[6] J. Cieslik, "On possibilities of the extension of Kharitonov's stability test for interval polynomials to discrete systems," IEEE Trans. Automat. Contr., vol. AC-32, pp. 237-238, Mar. 1987.

[7] A. C. Bartlett, C. V. Hollot, and L. Lin, "Root locations of an entire polytope of polynomials: It suffices to check the edges," Math. Contr., Signals, Syst., vol. 1, pp. 61-71, 1987

[8] M. Fu and B. R. Barmish, "Polytopes of polynomials with zeros in a prescribed region," in Proc. Amer. Contr. Conf., Atlanta, GA, 1988 .

[9] S. Bialas, "A necessary and sufficient condition for the stability of convex combinations of stable polynomials or matrices," Bull. Polish Academy of Sci., vol. 33, no. 9-10, pp. 473-480, 1985.

[10] M. Fu and B. R. Barmish, "Stability of convex and linear combinations of polynomials and matrices arising in robustness problems," in Proc. Conf. Inform. Sci. Syst., Johns Hopkins Univ., Baltimore, MD, 1987.

[11] F. Kraus, B. D. O. Anderson, E. I. Jury, and M. Mansour, "On robustness of low order schur polynomials," IEEE Trans. Circuits Syst., to be published.

[12] N. K. Bose, E. I. Jury, and E. Zeheb, "On robust Hurwitz and Schur polynomials," in Proc. IEEE Conf. Decision Contr., Athens, Greece, 1986.

[13] A. C. Bartlett and C. V. Hollot, "A necessary and sufficient condition for Schur invariance and generalized stability of polytopes of polynomials," IEEE Trans. Automat. Contr., vol. 33, pp. 575-578, June 1988.

[14] J. E. Ackermann and B., R. Barmish, "Robust Schur stability of a polytope of polynomials," IEEE Trans. Automat. Contr., vol. 33, pp. 984-986, Oct. 1988.

[15] E. Zeheb, "Necessary and sufficient conditions for root clustering in simple connected domains," IEEE Trans. Automat. Contr., to be published.

[16] M. K. Saridereli and F. J. Kern, “The stability of polynomials under 
correlated coefficient perturbations," in Proc. IEEE Conf. Decision Contr., Los Angeles, CA, 1987.

[17] A. Vicino, "Some results on robust stability of discrete time systems," IEEE Trans. Automat. Contr., vol. 33, pp. 844-847, Sept. 1988.

[18] S. P. Bhattacharyya, Robust Stabilization Against Structured Perturbations. New York: Springer-Verlag, 1987.

[19] S. Dasgupta and A. S. Bhagwat, "Conditions for designing strictly positive real transfer functions for adaptive error identification," in Proc. 24th Allerton Conf. Commun., Contr., Comput., Monticello, 1986

[20] K. S. Yeung and S. S. Wang, "A simple proof of Kharitonov's theorem," IEEE Trans. Automat. Contr., vol. AC-32, pp. 822-823, Sept. 1987.

[21] A. T. Fam and J. S. Meditch, "A canonical parameter space for linear systems," IEEE Trans. Automat. Contr., vol. AC-23, pp. 454-458, Sept. 1978.

[22] M. B. Argoun, "Stability of Hurwitz polynomials under coefficient perturbations: Necessary and sufficient conditions," Int. J. Contr., vol. 45 , no. 2 , pp. 739-744, Sept. 1987

[23] D. Hertz, E. I. Jury, and E. Zeheb, "Root exclusion from complex polydomains and some of its applications," Automatica, vol. 23, no. 3, pp. 399-404, 1987.

[24] M. Marden, Geometry of Polynomials (Mathematical Surveys, No. 3). Providence, RI: American Mathematical Society, 1966.

[25] L. A. Zadeh and C. A. Desoer, Linear System Theory. New York: McGraw-Hill, 1963.

[26] M. Saeki, "A method of robust stability analysis with highly structured uncertainties," IEEE Trans. Automat. Contr., vol. AC-31, pp. 935940 , Oct. 1986.

[27] R. R. E. de Gaston and M. G. Safonov, "Calculation of multiloop stability margin," IEEE Trans. Automat. Contr., vol. 33, pp. 156171, Feb. 1988
[28] A. Sideris and R. R. E. de Gaston, "Multivariable stability margin calculation with uncertain correlated parameters," in Proc. IEEE Conf. Decision Contr., Athens, Greece, 1986.

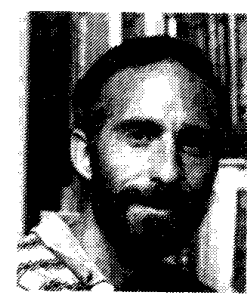

B. Ross Barmish (S'75-M'75) was born in Montreal, P.Q., Canada, in 1949. He received the bachelor's degree in electrical engineering from McGill University, Montreal, P.Q., Canada, in 1971, and the M.S. and Ph.D. degrees in electrical engineering from Cornell University, Ithaca, NY, in 1972 and 1975 , respectively.

From 1975 to 1978 he served as Assistant Professor of Engineering and Applied Science at Yale University, New Haven, CT. From 1978 to 1984 he was an Associate Professor of Electrical Engineering at the University of Rochester, Rochester, NY. In August of 1984 he joined the University of Wisconsin, Madison, where he is currently Professor of Electrical and Computer Engineering. His primary research interest lies in the area of systems and control.

Dr. Barmish has been involved in a number of IEEE Control Systems Society activities. From 1982 to 1984 he was a member of the Transactions Editorial Board and served as an Associate Editor for the IEEE TRANSACTIONS ON AUTOMATIC CONTROL. From 1983 to 1984 he served on the IEEE Control Systems Society Board of Governors and the Technical Activities Board. He is currently an Associate Editor for the journal Mathematics of Control, Signals and Systems. 